\title{
THE RELATIONSHIP BETWEEN PERSONALITY, SUBJECTIVE WELLBEING AND NARCISSISM AMONG COLLEGE STUDENTS
}

\author{
Najam ul Hassan Abbasi \\ Mushtaque Ali Channa**
}

\begin{abstract}
In order to study the relationship between personality, subjective well-being and narcissistic behavior of college students, a convenient sampling method was used to select college students, they were tested by Eysenck personality questionnaire (EPQ), total wellbeing scale and overt narcissism questionnaire (NPI16). The collected data were analyzed by t-test and correlation analysis. The findings suggest that there is a positive correlation between College Students' personality type (extraversion) and subjective wellbeing. College students with extraversion personality have high subjective well-being, while college students with introversion personality have low subjective well-being. There is a positive correlation between College Students' personality type (extraversion) and narcissistic behavior. College students with extraverted personality are more narcissistic, and college students with introverted personality are less likely to be narcissistic. There is a positive correlation between College Students' subjective well-being and narcissistic behavior. College students with high subjective well-being are more narcissistic, and college students with low subjective well-being are less likely to be narcissistic.
\end{abstract}

Keywords: College student; personality type; subjective well-being; Narcissism

\section{Introduction}

The current study intends to enrich the content of the relationship between personality, subjective well-being and narcissism. Previous studies have shown that extroverted individuals have higher subjective well-being. They are more stimulated by the outside world and produce happy emotions. Introverted individuals pay too much attention to their inner bad emotions, resulting in low subjective well-being. Extroverted individuals are more prone to narcissism. They are good at showing their advantages and treat their characteristics with confidence. They are usually charming in the process of interpersonal communication. Introverted individuals have less narcissistic behavior. They are more willing to pay attention to themselves, unwilling to compare with others, more gregarious, but not easy to influence others. There is also a correlation between subjective well-being and narcissistic behavior. When the individual's well-being is high, there will be more narcissistic behavior to show his happy heart. On the contrary, when individuals feel unhappy, they have less narcissistic behavior, less interpersonal communication

\footnotetext{
This work is Licensed under a Creative Commons Attribution-Non-Commercial 4.0 International License. $\infty$

* Najam ul Hassan Abbasi, Ph.D. Associate Professor of Psychology, Mianyang Normal University, Sichuan, China

** Mushtaque Ali Channa, MS. Scholar, Department of Psychology, University of Sindh, Jamshoro, Pakistan
} 
frequency, and pay more attention to their bad emotional experience. This study aims to analyze the relationship between College Students' personality, subjective well-being and narcissism, promote college students' mental health, help college students live and study better, and improve college students' ability to explore their potential and give full play to their advantages. It intends to help college students better adapt to college life. Narcissistic behavior may not only improve subjective well-being, but also affect others' evaluation of themselves. In order to enable college students to better manage their impression in the eyes of others, this study will analyze the causes of narcissistic behavior and provide ideas and methods for college students' impression management.

\section{Research Objective}

- To analyze the relationship between different types of personality \& subjective wellbeing

- To analyze the relationship between different types of personality \& narcissistic behavior

- To analyze the relationship between the subjective well-being $\&$ narcissistic behavior.

\section{Literature Review}

Personality is a kind of internal quality and a stable and unique psychological quality formed by individuals under the interaction of environment and heredity. So far, the psychological circle has not defined a clear concept of personality, and different psychological schools have different understandings of the concept of personality. Psychologists also express personality in different forms, such as personality types and personality traits.

Personality type theory holds that individual personality is divided into primary and secondary aspects, and personality can be divided into different types according to the main aspects of personality. Each personality type has great differences from each other. The concept of Introvert and extrovert personality was put forward by Jung, a psychologist of psychoanalysis school. He has conducted a lot of research and Analysis on these two types of personality, and believes that individuals with extraverted personality focus their interests and concerns on external objects, showing the characteristics of social love, active action, strong curiosity, openness and selfconfidence. Individuals with introverted personality tend to focus their interests and concerns on the interior of the subject, showing the characteristics of paying attention to their subjective world, like meditation, good introspection and so on.

Subjective well-being mainly refers to people's subjective judgment and subjective evaluation of their own living conditions and psychological feelings. Therefore, what determine people's subjective well-being are not only the actual events and real things, but also the individual's view of them. The book "related factors of subjective happiness" written by Wilson (1967) marks the beginning of formal research on subjective well-being in the field of psychology. At the same time, with the development and deepening of 
positive psychology, subjective well-being, as an important research object of positive psychology, has also been widely researched by psychologists ${ }^{1}$.

The first view of subjective wellbeing is that each individual's emotion is divided into two kinds, positive emotion and negative emotion. If the individual's positive emotion exceeds the negative emotion, the individual will experience more happiness. On the contrary, if the individual's negative emotion exceeds the positive emotion, the individual will experience little happiness. Subjective well-being is restricted and influenced by both positive and negative emotions ${ }^{2}$.

The second view is that subjective well-being is an individual's subjective cognition and subjective evaluation of their own life satisfaction. The representatives of this view are Neugarten and Cantril. They believe that subjective well-being is determined by individual cognition. Each individual interacts with his own environment. Therefore, objective existence is not an absolute factor affecting individual subjective well-being. Individual subjective cognition of objective existence can affect subjective well-being more. Individual life satisfaction is high, subjective well-being is correspondingly high, on the contrary, subjective well-being is low.

The third view is that subjective well-being is not only to experience happiness, but also to explore their own potential ${ }^{3}$. This view explains subjective well-being from the perspective of individual psychological development, and holds that the important influence of individual well-being is to make themselves develop effectively.

The fourth view holds that subjective well-being is an individual's overall evaluation of their own quality of life, and the standard for evaluating the quality of life is set by the individual. The overall evaluation of the quality of life reflects the individual's adaptation to the environment, so it has been supported and affirmed by many psychologists ${ }^{4}$.

Happiness has been the common pursuit of the general public since ancient times. From ancient Greek philosophers to modern psychological circles, the research on happiness has never stopped. There are many influencing factors of subjective well-being, mainly including genetic factors, family environmental factors, economic level factors, personality factors, work and study factors, social environmental factors and so on. Among them, there is interaction between personality and subjective well-being. Extroverted individuals tend to experience happy and positive events, and feel more subjective well-being. Neurotic individuals are pessimistic. They usually think that good events in life will not happen easily, but things always develop to the bad side. Therefore, there may be excessive tension, so neurotic individuals have low subjective well-being.

Chinese research on subjective well-being is also affected by different internal and external factors. In terms of family, the way children interact with their parents or other

\footnotetext{
${ }^{1}$ Wilson, "Related factors of subjective happiness", (1967)

${ }^{2}$ Bradburn, Norman M. "The structure of psychological well-being." (1969)

${ }^{3}$ Ryff, Carol D., and Corey Lee M. Keyes. "The structure of psychological well-being revisited." Journal of

Personality and Social Psychology 69, no. 4 (1995): 719.

${ }^{4}$ Diener, Ed, and Robert A. Emmons. "The independence of positive and negative affect." Journal of

Personality and Social Psychology 47, no. 5 (1984): 1105.
} 
caregivers in the early stage, and the parental rearing style will affect individual subjective well-being. Children who grow up in a free and democratic family are more likely to feel happy, and children who grow up in a harsh and cruel family atmosphere are not easy to feel happy under the influence of their original family. In terms of society, when the society develops well, the economic level is high, and the people live and work in peace and contentment, the individual happiness in the society is higher. On the contrary, the individual subjective happiness will decline when the society is turbulent. In terms of individual psychology, mental health has a great impact on well-being. Individuals with mental health, sound personality and independent thought have higher well-being. On the contrary, too many negative emotions will directly reduce subjective well-being. In order to improve subjective well-being, internal cause is the key. Improving one's own living standard, improving one's own current situation, establishing correct three outlooks and cultivating a strong heart can effectively improve individual subjective well-being and is conducive to the sustainable development of subjective well-being.

Freud believes that narcissism is a kind of psychological energy derived from libido. Individuals' early love is more towards themselves. With the growth of age, they gradually throw love outside the object. The child's main caregiver (usually the mother) has become the first object of the child's investment in love. If the child receives setbacks and obstacles in the process of outward investment in love, love will be reflected on the child himself. Such people will have secondary narcissism. They obviously regard themselves as the object of love, and the most extreme example is homosexuals ${ }^{5}$.

In 1976, Joffe and Sandler questioned Freud's view on narcissism, proposed "narcissistic disorder", studied narcissistic psychology and behavior from the perspective of clinical psychology, and believed that the emergence of narcissistic psychology and behavior should consider the impact of external environment on individuals. In 1968, the American Psychological Association defined narcissism as "focusing attention and interest on the subject itself"6.

The social cognitive theory of narcissism holds that the emergence of high narcissists is due to paying too much attention to themselves, thinking that they have talents different from ordinary people, are in the center of the crowd, and think that they are the leader of the group, like to influence or even dominate others. Social cognitive theory holds that high narcissists will elevate themselves by belittling others, think that they are mentally healthy and superior to others in many aspects. Sometimes high narcissists will have the phenomenon of overconfidence, unwilling to admit their mistakes and attribute their failure to the outside world. In serious cases, it will affect their mental health.

There are two forms of narcissism: explicit narcissism and implicit narcissism. Explicit narcissistic individuals need the attention of others and are eager to show their abilities. Explicit narcissists have great social charm and are easy to attract others' attention.

\footnotetext{
${ }^{5}$ Freud, Sigmund. "On narcissism: An introduction." In The Standard Edition of the Complete Psychological Works of Sigmund Freud, Volume XIV (1914-1916): On the History of the Psycho-Analytic Movement, Papers on Metapsychology and Other Works, pp. 67-102. 1957.

${ }^{6}$ Sandler, Joseph. "Dreams, unconscious fantasies and identity of perception." International Review of PsychoAnalysis 3 (1976): 33-42.
} 
Implicit narcissism is usually accompanied by inferiority complex. They pay too much attention to the negative evaluation of others, are particularly sensitive to the feedback of the outside world, and often feel dissatisfied. Implicit narcissists like to compare others with themselves, and usually focus on their own shortcomings, so they will feel inferior to others, resulting in inferiority or even self-abandonment. Individuals with high implicit narcissism are also prone to bad emotions and affect their mental health.

As the main body of society, man can create countless material and spiritual wealth. Everyone creates his own history in practical activities. But everyone is different. They have their own unique personality. There are no two identical people in the world. The uniqueness of personality means that a person's personality is formed under the interaction of heredity, maturity, environment, education and other pre acquired factors. Different genetic, survival and educational environments have formed their own unique psychological characteristics. People don't have exactly the same personality characteristics. Personality will also have an impact on individuals, which is the function of personality. Personality affects an individual's way of life and thinking to a certain extent, and sometimes even determines a person's destiny. Therefore, personality is an important factor affecting the success or failure of life.

With the continuous improvement of material living standards, people are increasingly pursuing spiritual satisfaction, one of which is the pursuit of happiness. Happiness is a very abstract word. Everyone has different definitions of happiness, so the concept of subjective well-being is formed. Individuals subjectively evaluate whether they are happy or not, and take this as a standard to measure individual well-being. Subjective well-being is a comprehensive psychological index. Its level depends not only on the external stimulation to the individual, but also on the individual's cognition and evaluation of the stimulation.

There is a correlation between narcissistic behavior and subjective well-being. On the one hand, narcissistic behavior may improve one's subjective well-being. Narcissistic behavior makes individuals feel satisfied and happy, which helps to improve individual subjective well-being. On the other hand, if individuals have high subjective well-being, they will have corresponding behavior. At this time, individual narcissistic behavior is likely to increase. Therefore, there is a correlation between narcissistic behavior and subjective well-being, and they affect each other.

\section{Research Methodology Participants}

In this study, a questionnaire survey was conducted among college students in different colleges of Sichuan, China. Using the convenient sampling method, 215 questionnaires were distributed, 202 were effectively recovered, and 13 questionnaires were invalid. In the valid questionnaires, 59 male subjects filled in, accounting for $30.8 \%$; 143 female subjects filled in, accounting for $69.2 \%$. There were 73 subjects with urban origin and 129 subjects with rural origin. There were 78 only child subjects and 124 non only child subjects. 


\section{Instruments \\ Eysenck Personality Questionnaire (EPQ)}

Eysenck Personality Questionnaire was used including four subscales. They are emotional scale, psychopathic scale, internal and external tendency scale and validity scale. Eysenck Personality Questionnaire has high reliability and validity. The revised version of Chinese still divides the scale into adult version and child version, but the number of items has been reduced, and still retains high reliability and validity.

This study mainly measures individual personality types, so only the subscale measuring internal and external tendencies of personality was intercepted, which was revised by Chinese psychologist Gong Yaoxian in 1983. The Chinese version has 21 questions in the revised version of the internal and external tendency scale. Each question has two options, namely "yes" and "no". The total score of the subjects is calculated according to the situation of the subjects filling in the questionnaire. If the total score is in the range of 114 , it is introverted, if the total score is in the range of 14-21, it is intermediate, and if the total score is in the range of $21-42$, it is extroverted.

\section{Overall well-being scale}

The overall well-being scale used to measure individual subjective well-being has high reliability and validity. It serves the compilation of the American Center for health statistics and is a good customized measurement tool. There are 33 items in the original scale, the average score of men is maintained at 75 points and that of women is maintained at about 71 points. The higher the total score, the higher the subjective well-being level of the subjects. The scale used in this study is a Chinese version revised by Duan Jianhua, with a total of 18 questions. This version retains the high reliability and validity of the original scale and is widely used in China, and its reliability and validity have been further verified.

\section{Narcissistic Personality Inventory (NPI)}

Narcissistic personality Inventory (NPI) is a common scale to measure narcissism, which has very high reliability and validity. There are 40 questions in total. Each question has two options, namely "yes" and "no". In this study, npi16 pairs of explicit narcissism questionnaire was used. Each question contained two options. Subjects chose one option in line with their real situation in each question. The questionnaire covers four dimensions: desire for power, sense of superiority, sense of privilege and self-admiration. Individuals with higher scores in the narcissism scale are more sensitive, have lower selfesteem, have more serious depression tendency, and may be filled with resistance.

\section{Procedure}

After taking the consent from the research participants, the participants were briefed on how to approach the scales. Once it was made sure that the participants have understood the instructions, they were handed over the scales and asked to return the scales in time. For the data processing and analysis, SPSS v. 20 was used, and the methods of independent sample t-test and correlation analysis were used for data analysis. 


\section{Results and analysis}

Analysis of the Correlation among study variables found that there was no significant difference in college students' personality (introversion extroversion) on gender, there was significant difference in the place of origin (rural-urban) $(p<0.01)$, and there was no significant difference in whether they were the only child ( See Table 1 for details).

There was significant difference in overall well-being between genders $(p<0.01)$, no significant difference in the place of origin (rural-urban), and no significant difference in whether they were only children ( See Table 1 for details).

There were significant differences in the four dimensions of overall well-being: energy, melancholy or happy mood, control of emotion or behavior, relaxation and tension ( $p<$ 0.05) ( See Table 1 for details).

The relaxation and tension of overall well-being were significantly different in whether they were only children $(p<0.05) \quad($ See Table 1 for details).

Explicit narcissism has no significant difference in gender, place of origin (urban rural) and whether it is an only child ( See Table 1 for details).

Table-1

General Status of Eysenck's Personality, overall Well-being and Explicit Narcissism

\begin{tabular}{|c|c|c|c|c|c|c|c|c|c|}
\hline Mkasmineatitens & $\begin{array}{l}\text { Atal } \\
\text { (n)-59) }\end{array}$ & $\begin{array}{l}F=a x \\
\text { in-LIy }\end{array}$ & t: & ${ }_{(n) 71)}$ & $\underset{t m=i \text { vo }}{\text { villege }}$ & t. & $\begin{array}{l}\text { Del: cial } \\
\text { (ain) }\end{array}$ & $\begin{array}{l}\text { Nim-0uly Chat } \\
\text { in }=121)\end{array}$ & 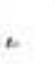 \\
\hline 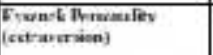 & $1911+2.77$ & 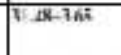 & IIII & $1567+13$ & $31-10+26 t$ & $206=$ & $5.75+25 x$ & i. $50-171$ & IRA \\
\hline 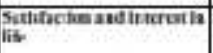 & $600+1.50$ & $\mid 15.90=15$ & iss & $1566=1$ A3 & $15,4 \pm 1,66$ & (4) & IS.n5:1.45 & $15.04=173$ & Q日is \\
\hline II:aith cescrom & 0.12 .247 & 7 BeLI.17 & De & $\sqrt{8.7 .2 .43}$ & 8054.13 & -1.49 & P., & 8.11 .2 .39 & -1.46 \\
\hline 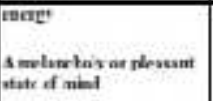 & $\begin{array}{l}1724254 \\
1575+1.56\end{array}$ & $\begin{array}{l}5.10=3 \pi \\
1155.7 \text { at }\end{array}$ & एक & 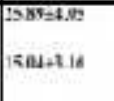 & $\begin{array}{l}26.06 \pm 1.91 \\
1104+15\end{array}$ & $\lim _{104}^{-3.32}$ & $\begin{array}{l}18645593 \\
1456+116\end{array}$ & $\begin{array}{l}20.70-394 \\
15.21-11\end{array}$ & $\begin{array}{l}-1.21 \\
-1 \text { जा }\end{array}$ \\
\hline 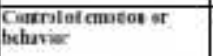 & $5.64 \pm 1.26$ & $24.53-20$ & $2 \pi T^{4}$ & $3 ., 4 \div \pm 2.12$ & $28.03+2.06$ & LA & B.13221 & $25.20=204$ & -6.57 \\
\hline 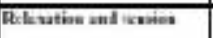 & 1751.128 & E.76.3.17 & $331^{+4}$ & 16113.16 & 16.361 .98 & $-1 .+8$ & $1572.2 \times 4$ & 16.62 .361 & $-1 . \mathrm{W}^{\prime}$ \\
\hline Orand writ-beint & II.,46E-11.11 & एवजयसाख़ा & $3 \mathrm{~N}^{2}$ & $15.2=113$ & T6.261055 & -1.21 & 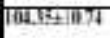 & 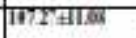 & -1.6 \\
\hline in ani for power & BI: $=87$ & 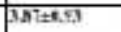 & 85 & $\overline{1.92+0.56}$ & $382=1.55$ & 475 & 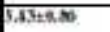 & $3.57 \pm 6.87$ & -6.50 \\
\hline 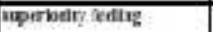 & $719=1.05$ & $753 \pm 1.13$ & -5.77 & $7,03=134$ & $7.4=1.11$ & Lat & $751=1.24$ & $75 E+17$ & Q8: \\
\hline Sente of prinkete & जिसाप & $5.94 \pm .15$ & Din & $0.18=9.94$ & $5.9 \pm 1.7 y$ & L.5 & $2.71 \pm 0.52$ & $5.94=1.01$ & 051 \\
\hline Selfadminatien & .12.0.01 & $207+6.5 x$ & in & 1.67 .650 & 1062.90 & 0.11 & Dowes & 0 BLE, & 015 \\
\hline Kspliát-Sastínzm & 11.25 .2 .7 & 2.78 .2 .92 & 231 & $11662 \pi$ & $2.16 \cdot 2.74$ & DIs & $11.32 .2 \pi$ & $2.32 \mathrm{n}$ & थः \\
\hline
\end{tabular}




\section{Relationship between Narcissism and Personality}

The research shows that there is a significant positive correlation between College Students' personality (extraversion) and overall well-being, and the correlation coefficient is 0.27 . College students with extrovert personality have a high overall well-being, while college students with introversion have a low overall well-being ( See Table 2 for details).

There is a significant positive correlation between College Students' personality (extraversion) and narcissism, and the correlation coefficient is 0.43. Extraversion college students are more likely to narcissism, while introversion college students are not easy to narcissism ( See Table 2 for details).

There is a significant positive correlation between College Students' subjective well-being and narcissism, and the correlation coefficient is 0.14 . College students with high subjective well-being are more narcissistic, and college students with low subjective wellbeing are less likely to be narcissistic ( See Table 2 for details).

Table-2

Correlation coefficients of Eysenck personality, overall Well-being and Explicit Narcissism

\begin{tabular}{|l|c|c|c|c|c|}
\hline & $M$ & $S D$ & $I$ & 2 & 3 \\
\hline $\begin{array}{l}\text { Eysenck Personality } \\
\text { (extraversion) }\end{array}$ & 31.67 & 3.69 & 1 & & \\
\hline Overall well-being & 106.14 & 11.01 & $0.27^{* *}$ & 1 & \\
\hline Explicit Narcissism & 21.34 & 2.70 & $0.43^{* *}$ & $0.14^{*}$ & 1 \\
\hline
\end{tabular}

\section{Correlation among dimensions of Eysenck's personality, overall well-being and explicit narcissism}

The research shows that the three dimensions of College Students' overall well-being, energy, melancholy or happy mood and control over emotion and behavior, are significantly correlated with the four dimensions of power desire, superiority, privilege and self-admiration in explicit narcissism ( See Table 3 for details). 
Table-3

Correlation coefficients of Eysenck personality, overall Well-being and Explicit Narcissism

\begin{tabular}{|c|c|c|c|c|c|c|c|c|c|c|c|c|}
\hline & a & 50 & 1 & 2 & 3 & 4 & 5 & s & 7 & - & $y$ & 10 \\
\hline Fymani Fermadily & $312 \mathrm{en}$ & 4 & 1 & & & & & & & & & \\
\hline 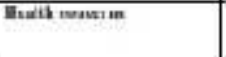 & 7.00 & 226 & ent & 1 & & & & & & & & \\
\hline wate & 1611 & 105 & num & $m+$ & $\mathbf{I}$ & & & & & & & \\
\hline 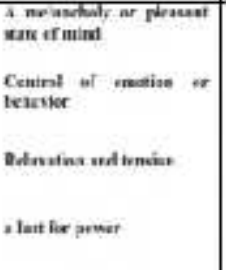 & 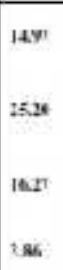 & sis & $\begin{array}{l}\text { 12: } \\
\text { ant } \\
\text { ant }\end{array}$ & $\begin{array}{l}\cos \\
\cos x \\
\cos \end{array}$ & Lar" & $\int_{0 \leqslant 1}^{1}$ & $\begin{array}{l}2.88 \\
131 \% *\end{array}$ & 214 & 1 & & & \\
\hline 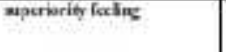 & 731 & H4 & as:- & $e n$ & 260 & 6.05 & $0, x^{t}=$ & w. & e.11 & 1 & & \\
\hline Seac af printigy & रथा & an & ant & 41 & en & a.1e & $11 x^{*}=$ & ait & $=x$ & ats & 1 & \\
\hline Vendmiration & sal & Bes & $2 y^{2}-$ & ent & 230 & 6.14 & $1.23^{+\infty}$ & nII & $238-$ & 24 & $024^{-}$ & 1 \\
\hline
\end{tabular}

\section{Discussion}

There is a significant positive correlation between College Students' personality (extraversion) and subjective well-being, which is consistent with the previous research results. College students with extroverted personality have higher subjective well-being, more cheerful and lively personality, extensive interests, good communication, often have many friends, do things more resolutely and decisively, dare to challenge and so on. Therefore, extroverted college students interact more actively with the environment and get positive feedback, which effectively improves their subjective well-being. College students with introverted personality pay more attention to themselves. They usually don't like talking and communication. They follow rules and regulations and are not good at revealing their true feelings, but they often pursue emotional support in the crowd, etc. Therefore, college students with introverted personality often focus on their own bad emotional experience, get less external positive feedback and lower subjective well-being.

There is a significant positive correlation between College Students' personality (extraversion) and narcissism. College students with extraversion personality are more narcissistic, and college students with introversion personality are less narcissistic. College students with extraverted personality pay attention to the outside world and are eager to get positive attention and positive feedback from the outside world. Therefore, they are more willing to show themselves and reveal themselves, and the frequency of narcissistic psychology or behavior will be more. The college students with introverted personality have less to the outside world and lower requirements for external emotional support. They pay more attention to their own views and views. Therefore, they do not 
like to discuss in the crowd, do not like to dominate or influence others, rarely show themselves, and the possibility of narcissistic psychology and behavior is very low.

There is a significant positive correlation between overall well-being and explicit narcissism. This shows that happy college students have more narcissistic behavior and unhappy college students have less narcissistic behavior. Narcissism here refers to explicit narcissism. Its main characteristics include longing to become the center and focus of the team, liking to be a leader and dominate others but not good at being led, liking adventure or stimulating activities, liking self-expression, self-disclosure and self-confidence. This is consistent with the research results. When individuals feel happy, they are more likely to make some narcissistic behaviors to adapt to their own subjective emotions. In contrast, when individuals feel unhappy, narcissistic behavior will decrease. Subjective well-being is regarded as an important factor affecting narcissistic behavior. Individuals with high subjective well-being are more likely to show narcissistic behavior to show themselves.

\section{Conclusion}

This study provides an important basis and Enlightenment for discussing the relationship between College Students' personality, subjective well-being and narcissistic behavior. College students with different personality types will feel different levels of well-being and narcissistic behavior. There is also a close relationship between College Students' overall well-being and narcissistic behavior. We should pay close attention to promoting the mental health of college students. Subjective well-being affects college students' daily emotional state to a great extent. Both school and society should guide college students to develop in a positive and healthy direction. Understanding the relationship among personality, subjective well-being and narcissistic behavior will help to improve the mental health level of college students.

\section{Bibliography}

Baoqiang, Zhang. 2012. Study on the relationship between parental rearing style, narcissistic personality and college students' aggression. Hangzhou Normal University, China.

Cheung, FM, W Fan, and C To. 2009. "Teaching and learning guide for: The Chinese Personality Assessment Inventory as a culturally relevant personality measure in applied settings." Social and Personality Psychology Compass (Wiley Online Library) 3 (6): 1113-1119.

Dan, he. 2016. The influence of parental rearing styles on Teenagers' online bullying: the role of narcissism and empathy. Central China Normal University, China.

Fengbo, Guo. 2017. Narcissistic personality regulates interpersonal cooperation decision-making and result evaluation. Tianjin Normal University, China. 
Fengbo, Guo, Zhang Zhen, Yuan Sheng, Jing Yiming, and Wang Yiwen. 2016. "Theoretical model and neurophysiological mechanism of narcissistic personality." Advances in psychological science 24 (08): 1246-1256.

Guihua, Wang, Zi Fei, and Li Yang. 2015. "The structure of narcissistic concept and its relationship with personality disorder tendency." Chinese Journal of clinical psychology 23 (6): 996-1002.

Hui, Huang. 2014. Research on the relationship between College Students' materialist values, gratitude and subjective well-being. Hunan Normal University, China.

Jiameng, Song, and Fan Huiyong. 2013. "Meta analysis of the relationship between social support and subjective well-being." Progress in psychological science 13 (21): 1357-1370.

Jieqing, Wu, Dong Yongyan, Xiong Junmei, and Cao Yu. 2016. "The relationship between College Students' initiative personality and career adaptability: the mediating role of achievement motivation and its gender differences." Psychological development and education 32 (05): 547-556.

Jiyue, Chen, Xu Jianping, Li Hongyan, Fan Yexin, and Lu Xiaolan. 2015. "Development and comparison of five factor oriented personality test." Progress in psychological science 23 (03): 460-478.

Lina, Song. 2015. Research on the relationship between college graduates' initiative personality, career adaptability and job selection anxiety. Inner Mongolia Normal University, China.

Liying, Jiao, Yang Ying, Xu Yan, Gao Shuqing, and Zhang Heyun. 2019. "Chinese good and evil: personality structure and connotation." Journal of psychology 51 (10): 1128-1142.

Lizhu, Yang. 2015. "Thirty years of research on personality development and cultivation of Chinese children and adolescents." Psychological development and education 31 (01): 9-14.

Ping, Zhang, and Wang Haibin. 2015. "The mediating role of College Students' emotional regulation self-efficacy between neuroticism, extraversion and subjective well-being." Chinese Journal of mental health 29 (02): 139-144.

Qian, Ding, Zhang Yongxin, Wei Hua, Niu gengfeng, and Zhou zongkui. 2016. "The relationship between narcissistic personality, selfie behavior and positive emotions of college students." Chinese Journal of clinical psychology 24 (03): 519-522.

Qing, Yang. 2016. Stress and subjective well-being of college students: the mediating role of psychological capital and the regulatory role of social support. Sichuan Normal University, China. 
Shufeng, Zhang, Ding Ling, Hu Dongmei, and Si Jiwei. 2016. "The relationship between College Students' independent personality and career decision-making difficulties: the mediating role of state trait anxiety." Chinese Journal of clinical psychology 24 (04): 684-688.

Songli, Mei, Chai Jingxin, and Guo Jinhua. 2015. "Adolescent subjective well-being and Internet addiction: the mediating role of self-esteem and self-control." Psychological development and education 31 (05): 603-609.

Tingting, Huang, Liu Liqian, Wang Dahua, and Zhang Wenhai. 2016. "Economic status and econometric status: the impact of social status comparison on subjective well-being and its age differences." Journal of psychology 48 (+): 1163-1174.

Wei, Ding. 2014. Research on the relationship between College Students' personality traits, social support and mobile phone dependence. Guangxi Normal University, China.

Weiwei, Sun. 2014. Analysis of mediating and regulating effects between gratitude and subjective well-being of adolescents. Wenzhou University, China.

Xiang, Xiao. 2014. The relationship between College Students' personality traits, mobile phone use motivation and mobile phone dependence. Hunan Normal University, China.

Xiaoyan, Zhao. 2016. Research on the relationship between College Students' subjective well-being, life events, cognitive emotion regulation and narrative group counseling intervention. Shanghai Normal University, China.

Xiujuan, Yang, Zhou zongkui, Liu Qingqi, and Niu gengfeng. 2017. "The relationship between narcissism and the use of social networking sites." Progress in psychological science 25 (9): 1552-1564.

Xue, Wang. 2015. The relationship between College Students' Narcissism and romantic relationship satisfaction: the role of self-esteem and empathy. Shaanxi Normal University, China.

Xueping, Shen, and Hu Shi. 2015. "The relationship between College Students' initiative personality and job search clarity: the mediating and regulating role of professional mission." Chinese Journal of clinical psychology 23 (01): 166-170.

Yaqian, Wang, Wang Li, and Xiang Guangfu. 2017. "College students' fifth year personality and subjective well-being: the mediating role of psychological elasticity." Chinese Journal of health psychology 25 (1): 112-116.

Yiwen, Wang, Fu Chao, Ren Xiangfeng, Lin Yuzhong, Guo Fengbo, Zhang Zhen, Huang Liang, Yuan Bo, and Zheng Yuwei. 2017. "Evaluation of the results of the trust game of narcissistic personality regulation." Journal of psychology 49 (8): 1080-1088. 
Yu, Zhang. 2015. Research on the relationship between narcissism and prosocial behavior: the role of self-improvement and success or failure feedback. Shaanxi Normal University, China.

Zhaojie, Deng, Huang Hai, GUI Yafei, Niu Luying, and Zhou Chunyan. 2015. "The relationship between College Students' mobile phone dependence and parental rearing style and subjective well-being." Chinese Journal of mental health 29 (01): 69-73.

Zhipeng, Tian, Liu Yong, and Teng Zhaojun. 2014. "Characteristics of boredom tendency of middle school students and its relationship with narcissism and subjective well-being." Chinese Journal of clinical psychology 22 (6): 1062-1067. 